\title{
MUC1 (episialin) expression in non-small cell lung cancer is independent of EGFR and c-erbB-2 expression and correlates with poor survival in node positive patients
}

Francesca Guddo, Alexandra Giatromanolaki, Michael I Koukourakis, Clotilde Reina, Antonio-Maurizio Vignola, George Chlouverakis, John Hilkens, Kevin C Gatter, Adrian L Harris, Giovanni Bonsignore

Institute of

Respiratory Pathophysiology, Consiglio Nazionale delle Ricerche, Palermo, Italy F Guddo

C Reina

A-M Vignola

G Bonsignore

Department of Radiotherapy/Oncology, Laboratory of Cancer Biology, and Department of Biostatistics, University Hospital of Iraklion-University of Crete, Iraklion, Crete, Greece

A Giatromanolaki M I Koukourakis G Chlouverakis

Department of Tumour Biology, The Netherlands Cancer Institute, Amsterdam, The Netherlands J Hilkens

Department of Cellular Science and ICRF Medical

Oncology Unit, Oxford Radcliffe Hospital, Headington, Oxford,

UK

K C Gatter

A L Harris

Correspondence to: Dr Michael I Koukourakis, Tumour and Angiogenesis Research Group, 18 Dimokratias Avenue, Iraklion 71306, Crete, Greece.

Accepted for publication 23 April 1998

\begin{abstract}
Aim-To examine tumour samples immunohistochemically for MUC1 (episialin), epidermal growth factor receptor (EGFR), and c-erbB-2, since the disruption of the cell-cell adhesion system by MUC1 and the c-erbB oncoprotein family is known to be important in the development of metastasis in human cancers.

Methods-93 tumour samples from patients with early stage non-small cell lung cancer treated with surgery alone were examined for episialin, EGFR, and c-erbB-2.

Results-Episialin depolarised expression did not correlate with any of the histopathological variables examined (T,N stage, grade, histology, Ki67 proliferation index). No correlation was observed between episialin and EGFR or c-erbB-2 expression. Survival analysis showed that episialin depolarised expression correlated with poor prognosis $(p=0.003)$, especially in squamous cell cases $(p=$ 0.0003). Episialin expression defined a group of patients with poor prognosis in the node positive category $(p=0.003)$. In multivariate analysis episialin was the most significant independent prognostic factor $(p=0.007)$, followed by $N$ stage $(p=$ 0.04).

Conclusions-Depolarised expression of episialin is associated with poor outcome in early stage non-small cell lung cancer. Despite the similar activity on the cadherin cell-cell adhesion system, the expression of episialin and c-erbB oncoproteins is likely to be activated within different pathogenic pathways. (F Clin Pathol 1998;51:667-671)
\end{abstract}

Keywords: lung cancer; episialin; c-erbB-2

For a cancer to invade adjacent structures and to migrate to distant organs, complex mechanisms are required. Angiogenic factor secretion by tumour and stromal cells, endothelial cell migration and vessel formation, and expression of proteins involved in the cell-cell and cell-matrix adhesion disruption or cell motility are essential for the tumour to grow, invade, and metastasise. ${ }^{12}$

The cadherin-catenin cell-cell adhesion system is well known to be involved in animal morphogenesis and the maintenance of normal adhesion between cells. ${ }^{3}$ The epidermal growth factor receptor (EGFR) and c-erbB-2 transmembrane oncoproteins (members of the erb oncoprotein family) interact with the cadherincatenin complex and may inhibit cell adhesion function, thus enhancing cancer cell invasive potential. ${ }^{4}$ Episialin, also known as MUC1 (or PEM, CA-15-3 antigen, or EMA) is another transmembrane protein shown in vitro to reduce E-cadherin mediated cell-cell adhesion by steric hindrance. ${ }^{6}$ Episialin is a glycoprotein expressed at the apical side of normal glandular epithelial cells. In cancer cells, depolarised expression throughout the entire cell surface has been observed.?

In this study we examined the depolarised expression of MUC1 glycoprotein immunohistochemically in a series of early stage operable non-small cell lung cancers. We report correlations with histopathological variables, Ki67 mitotic index, EGFR, and c-erbB-2, as well as survival.

\section{Methods}

We examined 93 tumour surgical samples from patients with early operable (T1,2-N0,1 staged $^{8}$ ) non-small cell lung cancer. All patients were treated with surgery alone, without radiotherapy or chemotherapy. Histological diagnosis, grading, and $\mathrm{N}$ stage was done on haematoxylin and eosin stained sections. Of these, 35 were adenocarcinomas and 58 squamous cell lung carcinomas. Follow up ranged from 45 to 74 months (median 62).

\section{EPISIALIN IMMUNOHISTOCHEMISTRY}

Episialin MUC1 expression was assessed on paraffin embedded material with the 214D 4 MoAb (IgG1) using an avidin-biotin complex immunoperoxidase technique. This antibody recognises the protein backbone of episialin. Sections were dewaxed and rehydrated, and treated for 10 minutes with $3 \% \mathrm{H}_{2} \mathrm{O}_{2}$ to limit endogenous peroxidase activity. Samples were then washed three times in $50 \mathrm{mM}$ Tris- $\mathrm{HCl} /$ $150 \mathrm{mM} \mathrm{NaCl}, \mathrm{pH} 7.6$ (TBS), and nonspecific binding was blocked in normal rabbit serum (30 minutes, 1:20; Dako, Copenhagen, Denmark) in TBS, and incubated with the monoclonal antibody 214D4 (mouse IgG1, diluted 1:32000) overnight at $4^{\circ} \mathrm{C}$. The sections were washed thoroughly in TBS and incubated with biotin conjugated rabbit 

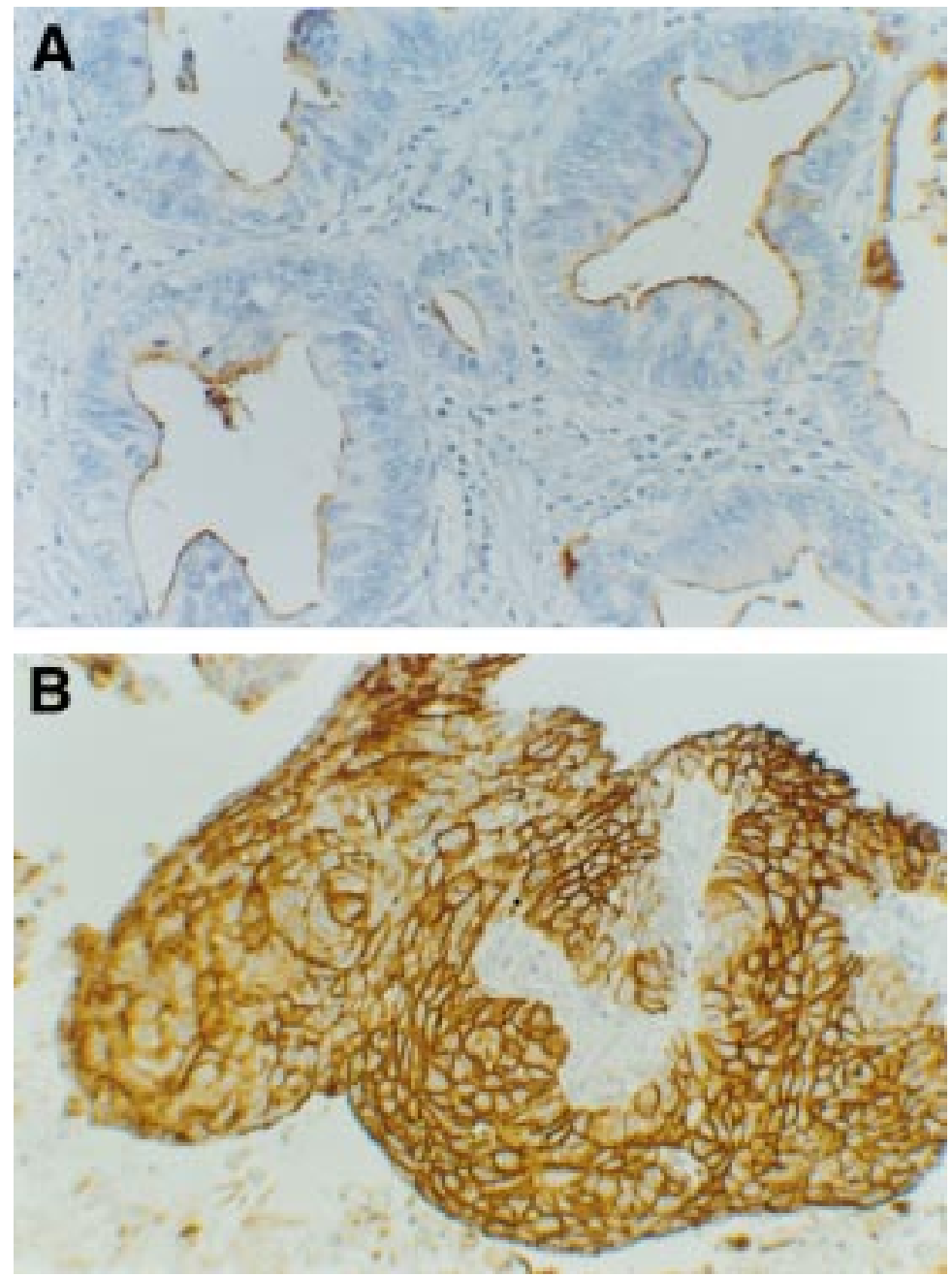

Figure 1 (A) A lung adenocarcinoma with polarised MUC1 expression. (B) A lung squamous cell carcinoma with depolarised circumferential cytoplasmic and membranous MUC1 expression.

antimouse immunoglobulin antibody (30 minutes, 1:200, Dako), followed by an avidinbiotin-peroxidase complex (30 minutes, $1: 100$, Dako). Finally, the sections were incubated with 3-4-3-4-diaminobenzidine as chromogen for five minutes and counterstained with haematoxylin. Omission of the primary antibody was used for negative control.

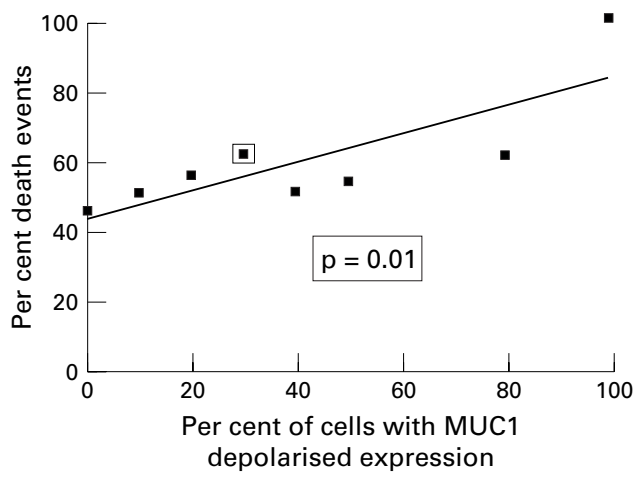

Figure 2 Linear regression of the percentage of deaths in sequential groups of patients defined by increasing cut off percentages of depolarised episialin expression in 93 non-small cell lung cancer patients.
In normal epithelium, episialin shows a polarised pattern of immunoreactivity. Positive "polarised pattern" of expression is defined when episialin is localised in the cytoplasmic vacuoles or in the cell membrane at the apical site of cells. The circumferential cytoplasmic and membrane immunoreactivity, never seen in normal cells, was recorded as "depolarised" episialin expression (fig 1). The percentage of cancer cells with episialin depolarised expression was recorded. This allowed analysis using MUC1 as a continues variable. Cases were also divided into two groups following the percentage of cells with depolarised expression, thus positive (depolarised expression in more than $25 \%$ of cells) and negative $(0-25 \%)$. The $25 \%$ used cut off point was the mean percentage of cells with episialin depolarised expression, but also the point that defined the group of patients with the highest percentage of deaths at the time of analysis (fig 2).

OTHER IMMUNOHISTOCHEMICAL STUDIES

C-erbB-2 oncoprotein expression was assessed with the monoclonal antibody NCL-CB11 (Novocastra Laboratories, Newcastle upon Tyne, UK), which recognises the internal domain of the c-erbB-2 protein amino acid sequence. Staining was done with a previously described indirect immunoperoxidase technique. ${ }^{9}$ For a positive control we used a breast carcinoma with 15-fold amplification of the c-erbB-2 gene and as a negative the primary antibody was omitted. In a previous study we showed that membrane staining was impossible to assess in non-small cell lung cancer. ${ }^{9}$ C-erbB-2 expression was separately assessed for cytoplasmic and membrane patterns of reactivity. Two groups were considered for cytoplasmic staining: the group with positive reactivity (strong staining intensity in

Table 1 Correlation between episialin depolarised expression and histological and patient variables in 93 non-small cell lung cancer cases

\begin{tabular}{|c|c|c|c|c|}
\hline Variable & & $\begin{array}{l}\text { Mean \% positive } \\
\text { cells (SD) }\end{array}$ & $95 \% C I$ & $p$ Value \\
\hline \multirow[t]{2}{*}{ Histology } & SC & $31.2(31)$ & 23 to 39 & \multirow[t]{2}{*}{0.81} \\
\hline & AC & $29.7(30)$ & 19 to 40 & \\
\hline \multirow[t]{2}{*}{ T stage } & $\mathrm{T} 1$ & $33.8(31)$ & 22 to 45 & \multirow{2}{*}{0.48} \\
\hline & $\mathrm{T} 2$ & $29.0(30)$ & 21 to 36 & \\
\hline \multirow[t]{2}{*}{$\mathrm{N}$ stage } & No & $30.3(30)$ & 22 to 38 & \multirow[t]{2}{*}{0.88} \\
\hline & N1 & $31.2(32)$ & 20 to 42 & \\
\hline \multirow[t]{2}{*}{ Grade } & $\mathrm{I} / \mathrm{II}$ & $28.0(32)$ & 18 to 37 & \multirow[t]{2}{*}{0.44} \\
\hline & III & 32.9 (29) & 24 to 41 & \\
\hline \multirow[t]{2}{*}{ Ki67 } & $\mathrm{L} / \mathrm{M}$ & $31.2(31)$ & 24 to 38 & \multirow[t]{2}{*}{0.67} \\
\hline & $\mathrm{H}$ & $27.2(29)$ & 12 to 43 & \\
\hline \multirow[t]{2}{*}{ c-erbB-2 } & neg/weak & $28.5(31)$ & 21 to 35 & \multirow[t]{2}{*}{0.31} \\
\hline & pos & 35.9 (29) & 23 to 48 & \\
\hline \multirow[t]{2}{*}{ EGFR } & neg & $36.6(36)$ & 18 to 54 & \multirow{2}{*}{0.57} \\
\hline & pos & $31.7(30)$ & 23 to 39 & \\
\hline \multirow[t]{2}{*}{ Age } & $<65$ years & $32.2(32)$ & 23 to 41 & \multirow[t]{2}{*}{0.55} \\
\hline & $>64$ years & $28.4(28)$ & 19 to 37 & \\
\hline \multirow[t]{2}{*}{ Sex } & Female & $38.5(32)$ & 22 to 54 & \multirow{2}{*}{0.23} \\
\hline & Male & $28.8(30)$ & 21 to 35 & \\
\hline
\end{tabular}

AC, adenocarcinoma; CI, confidence interval; SC, squamous cell carcinoma. 

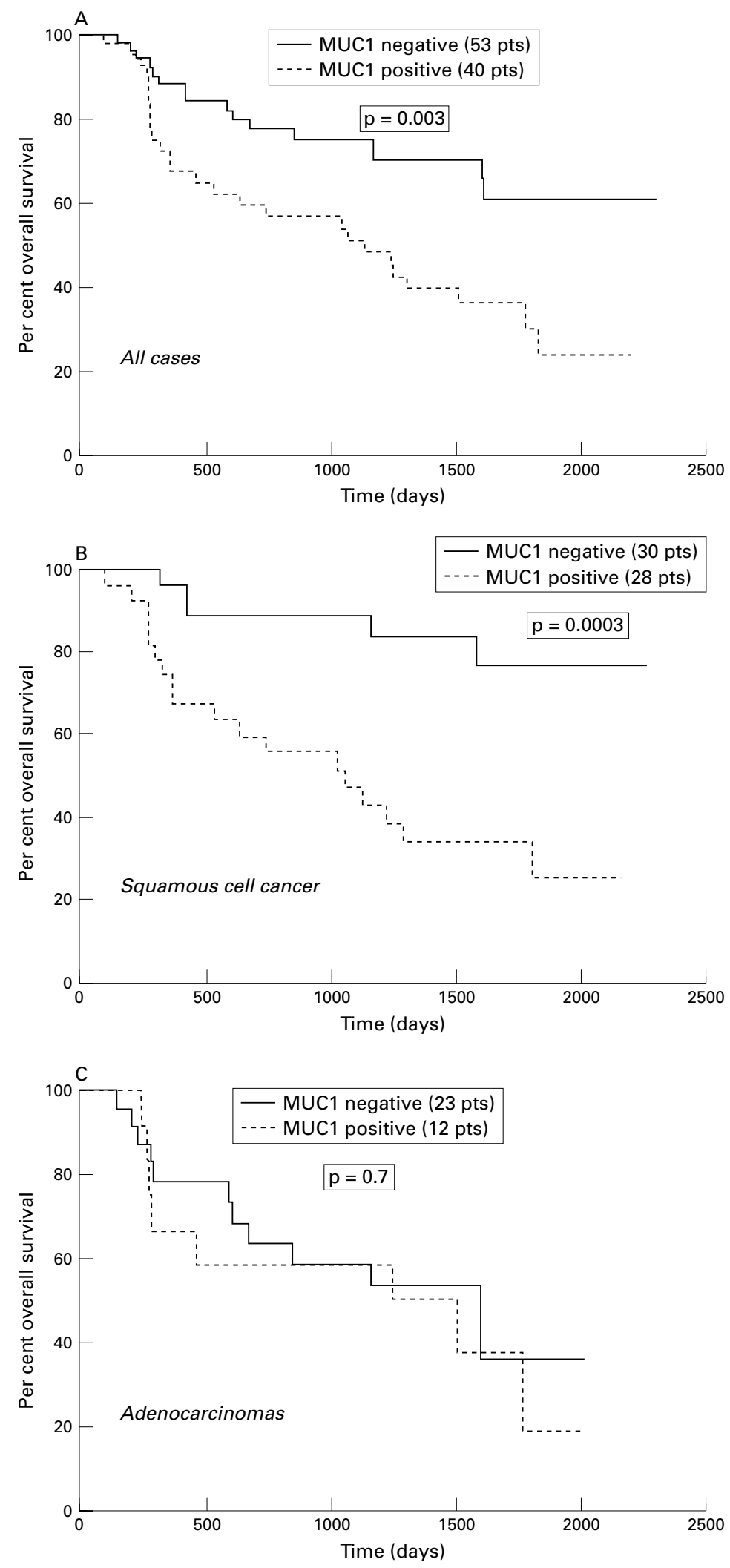

Figure 3 Kaplan-Meier survival curves for all cases (A), squamous cell cases (B), and adenocarcinomas $(C)$ according to MUC1 depolarised expression (positive $v$ negative).

$>50 \%$ of cells) and the group with negative or weak reactivity (all other cases). Membrane reactivity was positive when more than $10 \%$ of cells had a clearly defined membrane staining.
EGFR1 murine monoclonal antibody was used to identify EGFR by means of an indirect immunoperoxidase technique $^{10}$ on $5 \mu \mathrm{m}$ cryostat sections. Samples with negative $(-)$ or weak $(+)$ staining intensity were considered as negative, while moderately $(++)$ or strongly $(+++)$ positive were considered as positive.

Proliferative index was assessed with the monoclonal antibody Ki67. ${ }^{11}$ Frozen material was taken from two separate areas of the tumour and the Ki67 assessment was based on the average value. Three groups were considered, based on the percent of stained nuclei: $0-10 \%=$ low proliferative index, $10-40 \%=$ medium, and $>40 \%=$ high .

\section{STATISTICAL ANALYSIS}

Statistical analysis was performed using the Stata 3.1 Package (Stata Corporation, Texas, USA). An unpaired two tailed $t$ test was used for testing relations between categorical tumour variables. Survival curves were plotted using the Kaplan-Meier method, and the log-rank test was used to determine statistical differences between life tables. A Cox proportional hazard model was used to assess the effects of patient and tumour variables on overall survival. A probability $(\mathrm{p})$ value $<0.05$ was considered significant.

\section{Results}

Depolarised MUC1 expression in more than $25 \%$ of cancer cells was observed in 40 of 93 cases $(43 \%)$, where 28 of 58 squamous cell carcinomas (48\%) and 12 of 35 adenocarcinomas $(34 \%)$ showed depolarised patterns. Table 1 shows the correlation of MUC1 depolarised expression with histological and patient variables in 93 non-small cell lung cancer cases. No association was found with histology, nodal metastases, tumour differentiation, Ki67 proliferation index, patient age, or sex. No correlation of MUC1 with EGFR or cytoplasmic c-erbB-2 expression was observed. Analysis for membrane c-erbB-2 reactivity was impossible since only three of 93 cases $(3.2 \%)$ showed a clearly defined pattern of membrane staining.

Overall survival analysis showed that MUC1 depolarised expression was associated with a significantly worse prognosis $(p=0.003$; fig 3A). This mainly concerned the cases with squamous cell histology ( $\mathrm{p}=0.0003$; fig $3 \mathrm{~B}$ ), while no significant association with survival was observed in the adenocarcinomas ( $p=0.7$; fig 3C). Analysis within the N stage groups showed that MUC1 depolarised expression defined a group of node positive cases with significantly worse prognosis (fig 4, A and $\mathrm{B}$; $\mathrm{p}=0.009$ ). Multivariate analysis showed that MUC1 expression was the most significant independent prognostic factor $(b=0.85, S E=0.30, p=$ $0.004)$ followed by $\mathrm{N}$ stage $(\mathrm{b}=0.62, \mathrm{SE}=$ $0.30, \mathrm{p}=0.04)$. None of the remaining variables had an independent prognostic significance. 

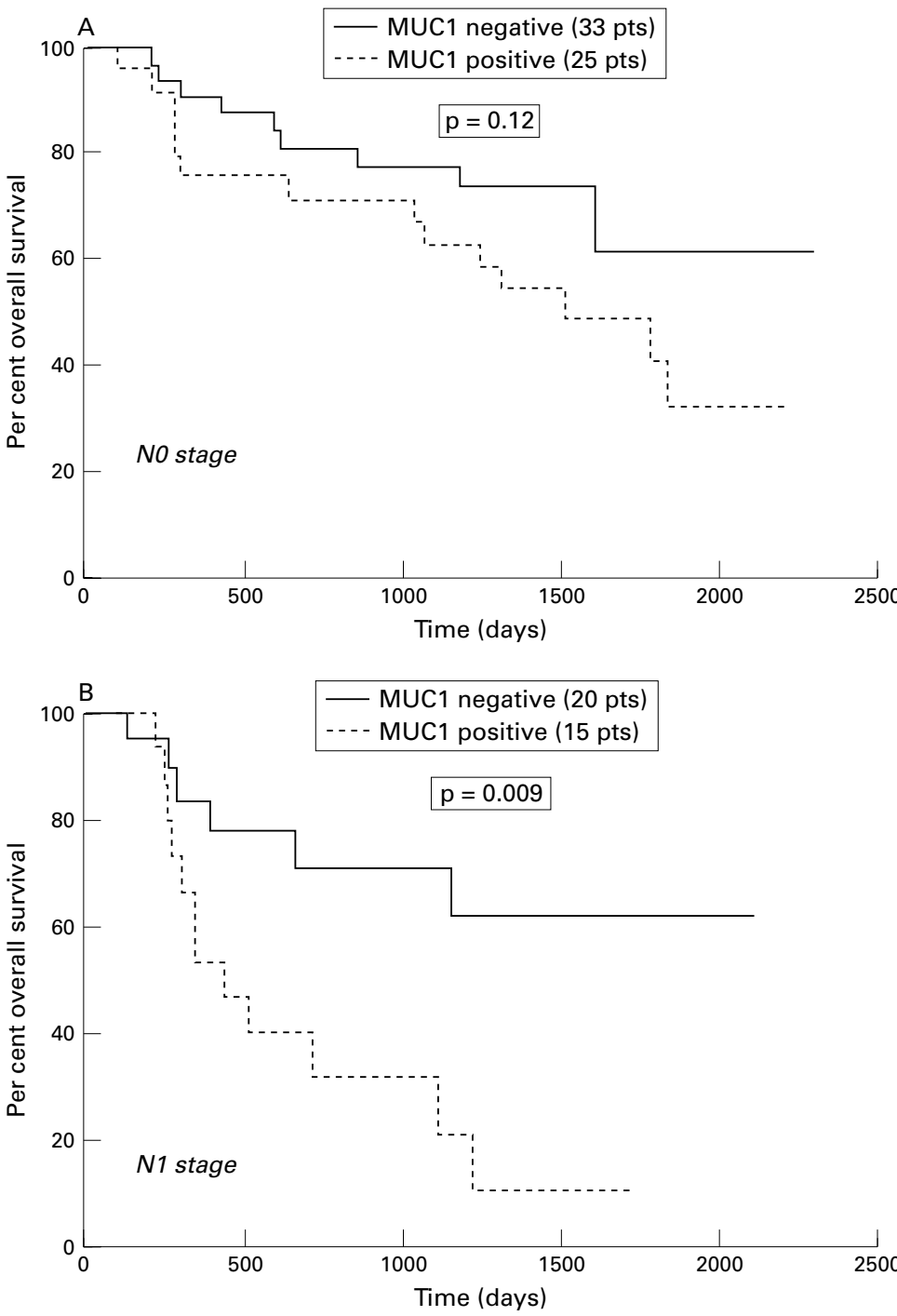

Figure 4 Kaplan-Meier survival cases for No stage (A) and N1 stage (B) groups according to MUC1 depolarised expression (positive v negative).

\section{Discussion}

Several in vitro studies suggest that MUC1 expression by cancer cells is an important component of biochemical events that enable metastasisation. Wesseling et al showed that MUC1 inhibits the E-cadherin mediated cellcell adhesion system, the length of MUC1 ectodomain being the dominant factor for inhibition to occur. ${ }^{6}$ High MUC1 levels also reduce the integrin mediated cell adhesion to the extracellular matrix. ${ }^{12}$ Although nonrestricted $\mathrm{T}$ cell cytotoxicity to the major histocompatibility complex elicited by MUC1 is reported, ${ }^{13}{ }^{14}$ MUC1 expressing cells are less susceptible to $\mathrm{T}$ cell mediated lysis, which may contribute to cancer cell escape from immune surveillance. ${ }^{15}$

In this study we examined immunohistochemically the depolarised expression of MUC1 in a series of non-small cell lung cancer patients treated with surgery alone. Depolarised MUC1 expression in more than $25 \%$ of cells was observed in about $45 \%$ of cases. High levels of MUC1 mRNA in lung cancer have been recently reported. ${ }^{16}$ In a study by Nguyen et $a l,{ }^{17}$ high concentrations of MUC1 mRNA were found in normal lung tissue and adenocarcinomas, while MUC1 levels were not increased in squamous cell cancer. In our study we examined the depolarised expression of MUC1 and found no difference between squamous cell carcinomas and adenocarcinomas. Disturbance of localisation rather than the mRNA expression level may more accurately reflect the aberrant function of the MUC1 membrane bounding mucin.

We also observed no correlation between MUC1 expression in lung cancer and histopathological variables including $\mathrm{N}$ stage, differentiation, and proliferation index. In breast cancer, ${ }^{18}$ depolarised MUC1 expression has been associated with node involvement and positive oestrogen receptors. However, no correlation of MUC1 with $\mathrm{T}$ stage or grade was observed. A significant association between MUC1 expression and node involvement has also been reported in colorectal cancer. ${ }^{19}$

The c-erb protein family is well known to disrupt the cadherin mediated cell adhesion system. In three studies of non-small cell lung cancer, c-erbB-2 expression was found to be associated with a worse outcome..$^{20-22}$ In a previous study we showed that c-erbB- 2 defined a poorer survival in cases with low vascularisation. ${ }^{23}$ Although membrane staining is generally accepted to be functionally relevant to tumour pathogenesis, only Kern's study ${ }^{20}$ was based on membrane staining. The studies by Tateishi et $a l,{ }^{21}$ Harpole et $a l,{ }^{22}$ and Giatromanolaki et $a l^{23}$ did not recognise a clearly defined pattern of membrane staining, the whole analysis been founded on cytoplasmic reactivity. Moreover, in a study by Kay et $a l{ }^{24}$ although membrane positivity was found in $7-21 \%$ of breast, bladder, and renal cases, no membrane reactivity was identified in squamous cell carcinomas, colon adenocarcinomas, and other tumours. Thus it seems that assessment of membrane reactivity cannot always be applied. Only $3.2 \%$ of our cases expressed a clearly defined pattern of c-erbB-2 membrane staining. Further staining of our cases with another c-erbB-2 antibody (Dako) using the APAAP technique confirmed that membrane staining assessment is not feasible in non-small cell lung cancer (data not shown). In the present study we examined possible cooperation of the MUC1 protein with the EGFR and c-erbB-2 oncoproteins. Although an unpublished study reported downregulation of MUC1 after transfection of mammary cancer cells with c-erbB- $2,{ }^{25}$ we failed to confirm a similar association. This may show that in lung cancer, although MUC1 and c-erbB proteins mediate cell-cell adhesion disruption, the regulation of their expression is controlled by different mechanisms.

Although several reports show a correlation of MUC1 expression with survival in breast, colon, and prostate cancer, ${ }^{18} 2627$ the role of MUC1 expression in lung cancer has not been clarified. A preliminary report from Japan in 38 non-small cell lung cancer cases revealed a possible association of MUC1 expression with poor survival. ${ }^{28}$ In our study, despite the absence of MUC1 association with 
histopathological variables, we observed a striking association of MUC1 depolarised expression with poor survival in node positive non-small cell lung cancer cases. Up to now nodal metastasis is considered the most important prognostic variable, and this was also confirmed in a previous study of our own. ${ }^{29}$ This is probably because node involvement is the best proof of cancer cell ability to migrate and form colonies. Why MUC1 expression in node positive cases correlates with poorer survival requires further investigation. Escape from immune surveillance ${ }^{15}$ could be an explanation, although a possible role of MUC1 in pure haematogenous spread cannot be excluded. Indeed, the interaction between MUC1 and ICAM-1 has recently been reported to be critical for the expression of bloodborne metastases in breast cancer. ${ }^{30}$ Our findings further support the existence of differences between pathogenic pathways that control lymphatic and bloodborne metastases.

We conclude that MUC1 is an important molecule, the depolarised expression of which relates to poor outcome in early stage nonsmall cell lung cancer. Although both MUC1 and c-erbB oncoproteins are involved in the disruption of cell-cell adhesion, it seems that their upregulation is triggered by nonoverlapping mechanisms which are activated within different pathogenic pathways.

This study was financially supported by the Imperial Cancer Research Fund (ICRF), Consiglio Nazionale dele Richerche (CNR), and the Tumour and Angiogenesis Research Group (TARG).

1 D'Amore P. Angiogenesis as a strategy for antimetastasis. Semin Thromb Hemost 1988;14:73-8.

2 Takeichi M. The cadherins: cell-cell adhesion molecules controlling animal morphogenesis. Development 1988;102: 639-55.

3 Scott PAE, Harris AL. Current approaches to targeting cancer using antiangiogenesis therapies. Cancer Treat Rev cer using antian

4 Kanai Y, Ochiai A, Shibata T, et al. c-erbB-2 gene product directly associates with beta-catenin and plakoglobin. Biochem Biophys Res Commun 1995;208:1067-72.

5 Ochiai A, Akimoto S, Kanai Y, et al. C-erbB-2 gene product associates with catenins in human cancer cells. Biochem Biophys Res Commun 1994;205:73-8.

6 Wesseling J, van der Valk SW, Hilkens J. A mechanism for inhibition of E-cadherin-mediated cell-cell adhesion by the membrane-associated mucin episialin/MUC1. Mol Biol Cell 1996;7:565-77.

7 Hilkens J, Buijs F, Hilgers J, et al. Monoclonal antibodies against milk-fat globule membranes detecting differentiation antigens of the mammary gland and its tumors. Int $\mathcal{F}$ Cancer 1984;34:197-206.

8 Mountain CF. A new international staging system for lung cancer. Chest 1986;89(suppl):225-33.

9 Giatromanolaki A, Gorgoulis V, Ranjan C, et al. c-erbB-2 expression in non small cell lung cancer. Anticancer Res 1996;16:987-94.
10 Veale D, Aschroft T, Gibson GJ, et al. Epidermal growth factor receptors in non-small cell lung cancer. Br f Cancer 1987;55:513-16.

11 Tungekar MF, Gatter KC, Dunnil MS, et al. Ki-67 immunostaining and survival in operable lung cancer. Histopathology 1991;19:545-50.

12 Wesseling J, van der Valk SW, Vos HL, et al. Episialin (MUC1) overexpression inhibits integrin-mediated cell adhesion to extracellular matrix components. $\mathcal{F}$ Cell Biol adhesion to extracel

13 Barnd DL, Lan MS, Metzgar RS, et al. Specific major histocompatibility complex recognition of tumor-associated mucins by human cytotoxic T cells. Proc Natl Acad Sci USA 1989;86:7159-64.

14 Jerome KR, Barnd DL, Bendt KM, et al. Cytotoxic T-lymphocytes derived from patients with breast adenocarcinoma recognize an epitope present on the protein core of a mucin molecule preferentially expressed by malignant cells. Cancer Res 1991;51:2908-12.

15 Weil-van Kemenade E, Ligtenber MJL, De Boer AJ, et al. Episialin (MUC1) inhibits cytotoxic lymphocyte-target cell interaction. F Immunol 1993;151:1-10.

16 Seregni E, Botti C, Lombardo C, et al. Pattern of mucin expression in normal and neoplastic lung tissues. Anticancer Res 1996;16:2209-13.

17 Nguyen PL, Niehans GA, Cherwitz DL, et al. Membrane bound (MUC1) and secretory (MUC2, MUC3, and MUC4) mucin gene expression in human lung cancer. Tumour Biol 1996;17:176-92.

18 McGuckin MA, Walsh MD, Hohn BG, et al. Prognostic significance of MUC1 epithelial mucin expression in breast cancer. Hum Pathol 1995;26:432-9.

19 Ajioka Y, Allison LJ, Jass JR. Significance of MUC1 and MUC2 mucin expression in colorectal cancer. F Clin Pathol 1996;49:560-4

20 Kern JA, Slebos RJC, Top B, et al. C-erbB-2 expression and codon $12 \mathrm{~K}$-ras mutations both predict shortened survival for patients with pulmonary adenocarcinomas. 7 Clin Invest 1994;93:516-20.

21 Tateishi M, Ishida T, Kohdono S, et al. Prognostic influence of the co-expression of epidermal growth factor receptor and c-erbB-2 protein in human lung adenocarcinoma. Surg Oncol 1994;3:109-13.

22 Harpole DH, Hendon JE, Wolfe WG, et al. A prognostic model of recurrence and death in stage I non-small cell lung cancer utilizing presentation, histopathology and oncoprotein expression. Cancer Res 1995;55:51-6.

23 Giatromanolaki A, Koukourakis M, O'Byrne K, et al. Non small cell lung cancer: C-erbB-2 correlates with low angiogenesis and poor prognosis. Anticancer Res 1996;16:381925 .

24 Kay EW, Barry Walsh CJ, Curran B, et al. C-erbB-2 mmunostaining: problems with interpretation. $f$ Clin Pathol 1994:47:816-22.

25 Sundqvist K. Differential expression of MUC1 mucin in human mammary epithelial cells transfected with c-erbB2. ICRF Annual Colloquium, poster 8, 1995.

26 Nakamori S, Ota DM, Cleary KR, et al. MUC1 mucin expression as a marker of progression and metastasis of human colorectal carcinoma. Gastroenterology 1994;106: 353-61.

27 Ng YK, Pickens CL, Dang SM, et al. Prognostic significance of increased immunodetectable MUC1 in prostate cancer. Proceeding of 88th Annual Meeting of the AACR, 1997:542.

28 Tsuda T, Mitsudomi T, Ohgami A, et al. Mucin gene expression is associated with the poor prognosis in human non-small cell lung cancer. Proceeding of 86th Annual Meeting of the AACR, 1995:214.

29 Giatromanolaki A, Koukourakis M, O’Byrne K, et al. Prognostic value of angiogenesis in operable non-small cell lung cancer. F Pathol 1996;179:80-8.

30 Regimbald LH, Pilarski LM, Longenecker BM, et al. The breast mucin MUCI as a novel adhesion ligand for endothelial intercellular adhesion molecule 1 in breast cancer. Cancer Res 1996;56:4244-9. 